\title{
The problem of reversals in assessing implicit sequence learning with serial reaction time tasks
}

\author{
Joaquín M. M. Vaquero • Luis Jiménez • \\ Juan Lupiáñez
}

Published online: 19 July 2006

(C) Springer-Verlag 2006

\section{Exp Brain Res (2006) DOI 10.1007/s00221-006-0523-6}

In the Introduction, first paragraph, last sentence and second paragraph, first sentence the reference citations were given incompletely. The correct sentences are given here.

This issue is currently analyzed in the field of "implicit learning" and it continues to raise controversy between those who defend the existence of a close relationship between learning and awareness
(Lovibond and Shanks 2002; Shanks and St. John 1994) and those who claim that unconscious learning processes do continuously affect human behavior (Cleeremans et al. 1998; Lewicki et al. 1997; Reber 1993).

Implicit learning has been studied in the laboratory using different experimental preparations (for reviews see Cleeremans et al. 1998; Seger 1994), but one of the most widely used at the moment is that of sequence learning in the context of serial reaction time (SRT) tasks (Nissen and Bullemer 1987).

The online version of the original article can be found at http://dx.doi.org/10.1007/s00221-006-0523-6

J. M. M. Vaquero $(\bowtie) \cdot J$. Lupiáñez

Departamento de Psicología Experimental y Fisiología del

Comportamiento, Facultad de Psicología,

Universidad de Granada, Campus Universitario Cartuja s/n,

18071 Granada, Spain

e-mail: joaquinm@ugr.es

J. M. M. Vaquero $\cdot$ L. Jiménez

Universidad de Santiago de Compostela,

Santiago de Compostela, Spain 